\title{
Serum Metabolomic Biomarkers of Dementia
}

\author{
Malahat Mousavi ${ }^{a}$, ${ }^{\text {Pär Jonsson }}{ }^{b}$ Henrik Antti ${ }^{b}$ Rolf Adolfsson ${ }^{c}$ \\ Annelie Nordin ${ }^{c}$ Jan Bergdahld, k Kåre Eriksson ${ }^{\mathrm{e}}$ Thomas Moritz $^{\mathrm{g}}$ \\ Lars-Göran Nilsson ${ }^{h, j}$ Lars Nyberg ${ }^{a, f, h}$ \\ Departments of ${ }^{a}$ Integrative Medical Biology, ${ }^{b}$ Chemistry, Computational Life Science \\ Cluster, ${ }^{c}$ Clinical Sciences, Psychiatry, ${ }^{\mathrm{d}}$ Psychology, ${ }^{\mathrm{e}}$ Occupational and Environmental \\ Medicine and ${ }^{\mathrm{f}}$ Radiation Sciences, Umeå University, ${ }^{9}$ Department of Forest Genetics \\ and Plant Physiology, Swedish University of Agricultural Sciences, and h Umeå Center for \\ Functional Brain Imaging, Umeå, 'Institute of Environmental Medicine, and ${ }^{j}$ Aging Research

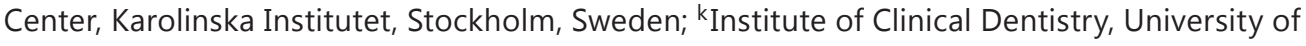 \\ Tromsø, Tromsø, Norway
}

\section{Key Words}

Memory · Early diagnosis - Alzheimer's disease - Vascular dementia - Gas chromatography

\begin{abstract}
Aims: This study compared serum metabolites of demented patients (Alzheimer's disease and vascular dementia) and controls, and explored serum metabolite profiles of nondemented individuals 5 years preceding the diagnosis. Methods: Cognitively healthy participants were followed up for 5-20 years. Cognitive assessment, serum sampling, and diagnosis were completed every 5 years. Multivariate analyses were conducted on the metabolite profiles generated by gas chromatography/time-of-flight mass spectrometry. Results: A significant group separation was found between demented patients and controls, and between incident cases and controls. Metabolites that contributed in both analyses were 3,4-dihydroxybutanoic acid, docosapentaenoic acid, and uric acid. Conclusions: Serum metabolite profiles are altered in demented patients, and detectable up to 5 years preceding the diagnosis. Blood sampling can make an important contribution to the early prediction of conversion to dementia.
\end{abstract}


Mousavi et al.: Serum Metabolomic Biomarkers of Dementia

\section{Introduction}

Alzheimer's disease (AD) is characterized by gradual progression of cognitive and behavioral changes. Much attention has been directed at identifying sensitive and specific biomarkers of the disease, with the hope that these will enable an earlier and more accurate diagnosis. There is abundant evidence that imaging [1-3] and cerebrospinal fluid (CSF) [4-6] markers are potent, and an influential model of dynamic biomarkers of the AD pathological cascade includes 4 imaging and CSF biomarkers [7].

The potential contribution of additional biomarkers for the early diagnosis of AD, notably the ones that require less invasive sampling methods, is under evaluation. One candidate approach is blood sampling, which has been used to study plasma [8,9] and determine serum metabolomic profiles in $\mathrm{AD}$ patients and in individuals progressing to $\mathrm{AD}$ [10]. A similar approach has been presented for saliva samples [11], although comparisons of saliva and serum analyses indicated limitations in using saliva for biomarker discovery [12].

The current prospective population-based study explored serum metabolite profiles, generated by gas chromatography/time-of-flight mass spectrometry (GC/TOFMS), of AD patients, patients with vascular dementia (VaD), and matched controls. Previous studies found some signs of metabolome alterations in individuals progressing to $\mathrm{AD}[10,13]$. We therefore explored the serum metabolite profile of individuals up to 5 years preceding the dementia diagnosis. Saliva samples were also available of the same participants at the same sampling occasion, which allowed further evaluation of the utility of saliva for biomarker discovery in dementia.

\section{Materials and Methods}

\section{Study Overview and Participants}

A total of 2,528 individuals in 3 samples (S1, S3 and S5) from the prospective, longitudinal Betula study [14] were initially enrolled in the study at its first (T1), second (T2) or fourth (T4) test wave, respectively (fig. 1a). At baseline, the participants ranged in age between 35 and 85 years. By design, all participants were cognitively normal individuals who were randomly selected from the population registry and followed over time to investigate agerelated cognitive development over time and early signs of dementia. At baseline and subsequently at 5-year intervals, the participants took part in a health assessment, which also included the collection of biological samples. The sampling of serum was carried out at each test wave (T1-T5), taken at random over the day, in a nonfasting condition. The blood samples were kept at refrigerator temperature $\left(+4^{\circ} \mathrm{C}\right)$ up to 1 day before centrifugation $(2,800 \mathrm{rpm}$ in $15 \mathrm{~min}$ ) and thereafter aliquoted into 2 - $\mathrm{ml}$ cryovials prior to long-time storage at $-80^{\circ} \mathrm{C}$. The saliva samples were only collected at test waves 4 and 5 in series of 4 to allow analysis of a 24 -hour curve $(07.00,11.00,16.00,23.00)$. The participants spit into a new plastic tube at each time point. During and after the saliva sampling, which took place in the subjects' home, the saliva was kept at refrigerator temperature $\left(+4^{\circ} \mathrm{C}\right)$ up to 1 day prior to being handed to the research staff. The samples were thereafter stored at $-20^{\circ} \mathrm{C}$. Within 1 week after the health assessment, a comprehensive neuropsychological battery was administered with several measures of episodic memory functioning (see below). The participants in the present metabolomic study were selected from the Betula study's forth test wave (T4) provided that both saliva and serum samples were available. The availability of saliva samples from participants with a manifest dementia disorder at T4 was limited, due to the fact that the collection of saliva required that the person himself/herself or with help could follow the procedure for sampling. Of the 1,842 individuals from the cohorts S1, S3 and S5 participating 
Mousavi et al.: Serum Metabolomic Biomarkers of Dementia

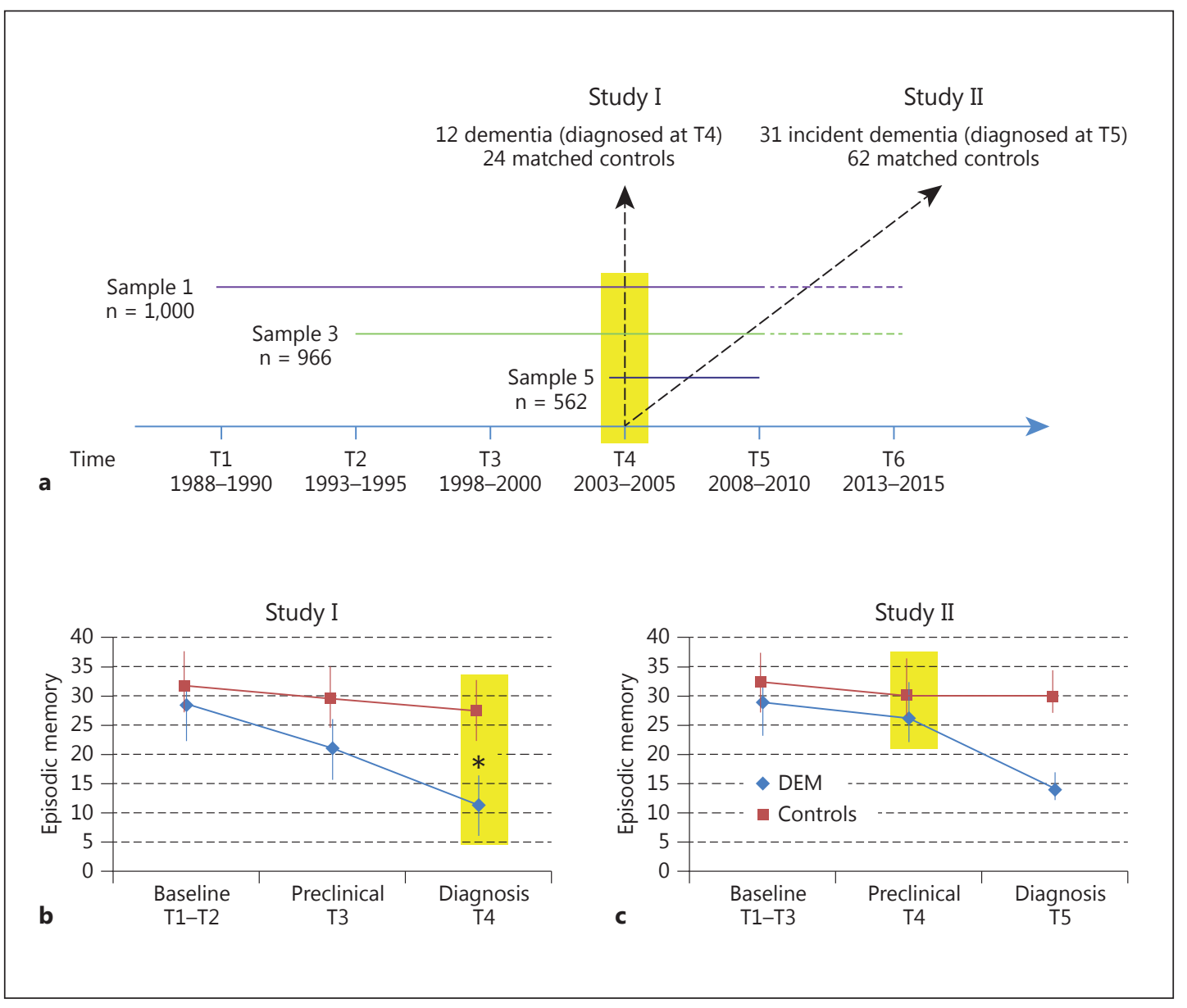

Fig. 1. Overview of the study design and patterns of episodic memory change. a The participants were included in the study at different test waves (T) and followed longitudinally. Serum and saliva samples were collected at T4 (yellow contour). The $n$ values refer to T4. b Episodic memory performance (mean; 1 SD) for demented and control participants in study I. The demented patients were diagnosed at T4, when they performed at a significantly lower level than controls. c Episodic memory performance (mean; 1 SD) for incident demented and control participants in study II. The demented patients were diagnosed at T5, 5 years after serum and saliva collection. They performed at a significantly lower level than controls at the time for diagnosis but not at the time for collection of biological samples. Sample 1 participants entered the study at T1, sample 3 participants at T2, and sample 5 participants at T4, so $\mathrm{n}$ varies across time points. Yellow shading indicates the time point when serum and saliva samples were collected. ${ }^{*} \mathrm{p}<0.05$ according to $t$ tests.

at T4, 12 participants met the criteria of being demented at T4 (9 AD; $3 \mathrm{VaD}$ ) and having both serum and saliva samples accessible. These individuals, along with age-, gender- and education-matched controls $(n=24)$, constituted study I. In study II, aimed at studying preclinical metabolomic biomarkers, the cases were defined as nondemented at $\mathrm{T} 4$ but with a progress towards dementia at T5. Study II comprised 31 cases (15 AD; $12 \mathrm{VaD} ; 4$ other) and 62 age-, gender- and education-matched controls. Thus, the majority of the included patients had AD (56\%) and an additional 35\% was classified as having VaD. The remaining 4 patients (9\%; all in study II) met the diagnostic criteria for other dementia subtypes, including dementia with Lewy bodies. The local ethics review board at Umeå University approved the study, and written informed consent was obtained from all participants. 


\section{Dementia Diagnosis}

During the relevant Betula study data collection period (1988-2010), repeated prospective and retrospective analyses of the dementia status were performed on all participants at baseline and every 5 years in order to identify diseased participants, ensure the accuracy of previously ascertained dementia diagnoses, and determine the year at which an individual fulfilled the core criteria for dementia, i.e. when cognitive symptoms became sufficiently severe to interfere with social functioning and instrumental activities of daily living [15]. AD and VaD were diagnosed using the DSM-IV criteria [16]. The more detailed NINCDSADRDA criteria for probable AD [15] and cognitive and neurological symptoms of vascular complications were also taken into account for the diagnosis of $\mathrm{VaD}$ [17].

A research geropsychiatrist coordinated the diagnostic evaluation and was responsible for the final diagnoses throughout the study period. At test waves T1, T2, and T3, up to 4 additional geropsychiatrists participated in the evaluation process, thereby establishing a solid ground for a diagnostic consensus.

The observations made at the health and cognitive evaluations formed the basis for a decision on performing a more extended diagnostic evaluation. The following predetermined criteria were used as a crude guide for further evaluation: Mini-Mental State Examination score $\leq 23$, a decline in cognitive performance compared to a previous test occasion (from high to average/low or from average to low), a decline in daily functional activities, a subjective loss of memory function expressed in the semi-structured interview, and any other deviations noticed by the testing team. The results obtained at clinical examinations, retrieved from medical records, were important collateral information and taken into account in the diagnostic decision process.

In 2011, an extensive quality assurance of the dementia diagnoses was performed in that the medical records of those with an established dementia diagnosis were blindly re-evaluated with regard to dementia status, subtype, and age at onset. A comparison between the previously diagnosed subtypes and the newly proposed ones was made, with the result that approximately $95 \%$ of the diagnoses were congruent with the previously given subtype diagnoses. In cases where the diagnoses were inconsistent, it was evident that the re-evaluated diagnoses were more reliable due to the longer time of follow-up. The diagnostic evaluation resulted in that 444 of a total of 4,069 individuals from all Betula cohorts (S1-S5) had developed a dementia disorder, with a clear preponderance of AD and VaD. Furthermore, the re-evaluation showed that only $19(0.4 \%)$ of all 4,445 Betula participants were improperly included in the study (i.e. these 19 were already demented at the time for inclusion), which provides strong support for the diagnostic evaluation method used.

\section{Episodic Memory Composite}

The episodic memory measure was a composite of five test variables: (1) immediate free recall of 16 visually and verbally presented short sentences, (2) delayed cued recall of nouns from the previously presented sentences, (3) immediate free recall of 16 enacted sentences, (4) delayed cued recall of nouns from the enacted sentences, as well as (5) immediate free recall of a list of 12 verbally presented nouns. Two alternate sets of sentences and nouns were used in 1-4, and four sets were used in 5, across measurement occasions. A full description of procedures and tests has been given previously [14]. The episodic memory composite score can a priori range between 0 and 76, with a higher score indicating better cognitive function [18]. Analyses of the internal consistency of the composite revealed a good level (Cronbach's alpha $=0.83$ ), and the test-retest reliability was estimated to be 0.79 (Pearson correlation) [19]. 


\section{Metabolite Extraction and GC/TOFMS Analysis of Serum and Saliva Samples}

Metabolite extraction from the serum samples was essentially done as previously described [20] by adding a 900- $\mu$ l methanol/water extraction mix [90:10 v/v; including 11 isotopically labelled internal standards $(7 \mathrm{ng} / \mu \mathrm{l})$, L-proline $-{ }^{13} \mathrm{C}$, succinic acid- ${ }^{13} \mathrm{C}$, salicylic acid-D6, Di-Na- $\alpha$-ketoglutarate- $-{ }^{13} \mathrm{C}$, L-glutamic acid- ${ }^{13} \mathrm{C}$, putrescine-D4, myristic acid- ${ }^{13} \mathrm{C}$, D-glucose- ${ }^{13} \mathrm{C}$, hexadecanoic acid- ${ }^{13} \mathrm{C}$, sucrose $-{ }^{13} \mathrm{C}$ and cholesterol-D7] to $100 \mu \mathrm{l}$ of serum. This was followed by rigorous agitation at $30 \mathrm{~Hz}$ for $2 \mathrm{~min}$ in a bead mill (MM 400; Retsch $\mathrm{GmbH}$, Haan, Germany), storage on ice for $120 \mathrm{~min}$ and $10 \mathrm{~min}$ of centrifugation at $14,000 \mathrm{rpm}$ at $4{ }^{\circ} \mathrm{C}$ (Centrifuge 5417R; Eppendorf, Hamburg, Germany). Two hundred microliters of each supernatant were transferred to GC vials and evaporated until dry in a speedvac (miVac, Quattro concentrator; Barnstead Genevac, Ipswich, UK). Derivatization was done in two steps: (1) methoxyamination, by adding $30 \mu \mathrm{l}$ methoxyamine in pyridine $(15 \mu \mathrm{g} / \mu \mathrm{l}), 10 \mathrm{~min}$ of shaking and $60 \mathrm{~min}$ of heating at $70^{\circ} \mathrm{C}$, was carried out for $16 \mathrm{~h}$ (at room temperature) and (2) trimethylsilylation, by adding $30 \mu \mathrm{l}$ MSFTA (N-methyl-N-trimethylsilyl-trifluoroacetamide) $+1 \%$ TMCS (trimethylchlorosilane), was carried out for $1 \mathrm{~h}$ (at room temperature). In the end, $30 \mu \mathrm{l}$ heptane, including methyl stearate $(15 \mathrm{ng} / \mu \mathrm{l})$, as an injection standard, was added.

Samples were then injected in a splitless mode by a CTC Combi Pal autosampler (CTC Analytics AG, Zwingen, Switzerland) into an Agilent 6890 gas chromatograph equipped with a $10 \mathrm{~m} \times 0.18 \mathrm{~mm}$ (internal diameter) fused silica capillary column with a chemically bonded 0.18- $\mu \mathrm{m}$ DB 5-MS stationary phase (J\&W Scientific, Folsom, Calif., USA). The column effluent was introduced into the ion source of a Pegasus III time-of-flight mass spectrometer, GC/ TOFMS (Leco Corp., St Joseph, Mich., USA). For the saliva samples, a similar procedure was used, with the difference that prior to the step of the methanol/water extraction, metabolites in the samples were concentrated by centrifuging $1,000 \mu \mathrm{l}$ of each saliva sample at 14,000 $\mathrm{rpm}\left(4^{\circ} \mathrm{C}\right)$ for $10 \mathrm{~min}$. Then $900 \mu \mathrm{l}$ of the supernatant was freeze-dried and the pellet was solved in 1,000 $\mu \mathrm{l}$ of methanol/water (7:3) including the same 11 internal standards.

\section{Data Processing and Identification of Metabolites}

The data extracted from GC/MS were processed using hierarchical multivariate curve resolution as previously described $[21,22]$. Resolved metabolite profiles were identified by comparing retention indices and mass spectra with data in retention index and mass spectral libraries [23], using NIST MS-Search v. 2.0.38 and an in-house mass spectral library database, the mass spectral library maintained by the Max Planck Institute in Golm (http://csbdb. mpimp-golm.mpg.de/csbdb/gmd/gmd.html), or the NIST98 mass spectral library. Retention index was calculated by relating retention time to an analytically characterized alkane series $\left(\mathrm{C}_{10}-\mathrm{C}_{40}\right)$. Normalization of the data was carried out according to Redestig et al. [24].

\section{Statistical Analysis of Data from Metabolomics}

Acquired and processed GC/TOFMS data were subjected to multivariate analysis and modeling using the SIMCA-P 13.0.02 software (Umetrics, Umeå, Sweden). The data sets were mean-centered and scaled to unit variance before analyses using OPLS-DA to detect and statistically verify differences between predefined sample classes (demented vs. controls) based on the whole resolved GC/TOFMS metabolic profile following an iterative variable selection procedure using the OPLS-DA variable importance plot. Variable importance plot values $>1$ was retained for further modelling and a maximum of two iterations were allowed. Inspection of individual profiles was used to screen for potential differences between dementia subtypes (AD, VaD). Multivariate analysis and univariate analysis by means of ANOVA were performed using the Statistical Toolbox (version 8.3) for Matlab (MATLAB 8.2.0.701; The MathWorks Inc., Natick, Mass., 2013). Differences in memory performance were evaluated by means of independent-samples $t$ tests (performed by SPSS). 
Table 1. Participant characteristics at the time (T4 in fig. 1) for the collection of serum and saliva for the samples examined in study I and II

\begin{tabular}{|c|c|c|c|c|}
\hline \multirow[t]{2}{*}{ Variables } & \multicolumn{2}{|l|}{ Study I } & \multicolumn{2}{|l|}{ Study II } \\
\hline & demented & control & demented & control \\
\hline Age, years & $75.0 \pm 4.2$ & $74.5 \pm 4.6$ & $65.3 \pm 9.1$ & $65.6 \pm 9.0$ \\
\hline Gender, $\%$ female & 67 & 67 & 68 & 68 \\
\hline MMSE score $(\max$ score $=30)$ & $24.0 \pm 3.2$ & $26.6 \pm 1.8$ & $27 \pm 2.1$ & $27.4 \pm 1.7$ \\
\hline APOE $\varepsilon 4$ carrier, $\%$ & 83 & 21 & 48 & 19 \\
\hline Education, years & $7.5 \pm 1.4$ & $7.7 \pm 1.4$ & $8.0 \pm 2.8$ & $8.1 \pm 2.6$ \\
\hline Total number per sample & 12 & 24 & 31 & 62 \\
\hline
\end{tabular}

The genotyping of APOE was done for all participants in study I and for $77 \%$ of the participants in study II. The values are stated as mean \pm 1 SD. MMSE $=$ Mini-Mental State Examination.

\section{Results}

In the first set of analyses (fig. 1a; study I), a total of 12 individuals from S1 and S3 with a dementia diagnosis at the time of the 4 th test wave were identified having both saliva and serum samples accessible ( 9 AD; 3 VaD). Two age-, sex-, cohort- and education-matched controls were identified for each patient. The 24 controls remained nondemented 5 years later at the 5th test wave. For the second set of analyses (fig. 1a; study II), 31 individuals were identified who were nondemented at the 4 th test wave, but received a diagnosis by the time of the 5 th wave (15 AD; $12 \mathrm{VaD} ; 4$ other). Thus, the serum and saliva samples were collected up to 5 years preceding the diagnosis for these individuals. Two age-, sex-, cohort- and education-matched controls were selected for each incident case $(n=62)$. Table 1 shows participant characteristics for the groups in each study.

Figure $1 \mathrm{~b}$ illustrates longitudinal memory change for demented patients and controls (study I). The yellow shading indicates the point in time when serum and saliva samples were collected. At baseline, 10-15 years preceding the diagnosis, the groups displayed comparable performance. A difference in performance began to emerge 5 years prior to the diagnosis (preclinical phase), and a marked difference was apparent at the time of the diagnosis. The corresponding data on memory change for incident cases with controls are shown in figure 1c (study II). At the preclinical phase, only a weak trend towards a group difference in performance was seen, whereas a marked group difference in memory performance was apparent at the time of diagnosis.

Serum metabolite profiles were compared between demented patients and controls (study I). The multivariate analysis yielded a significant model $(\mathrm{Q} 2=0.46, \mathrm{p}=0.0001 ; 68$ putative metabolites), which differed between demented patients and controls (fig. 2a).

The corresponding analysis of differences between incident cases and controls (study II) also revealed a group separation (fig. $2 \mathrm{~b}$ ). Although this model was not as strong as the one in study I, it was still significant according to the multivariate analysis $(Q 2=0.14, p=0.003$; 25 putative metabolites; 1 control was an outlier and was removed from the analysis).

To assess whether any metabolites contributed to the group separation in both study I and II, an ANOVA was conducted on all 208 putative metabolites to find metabolites that showed significant differences between demented/incident demented persons and controls (i.e. across both studies). Three metabolites were identified that contributed to the group separation in both studies: 3,4-dihydroxybutanoic acid, docosapentaenoic acid, and uric acid. They were upregulated for both diagnosed and incident demented cases relative to controls 


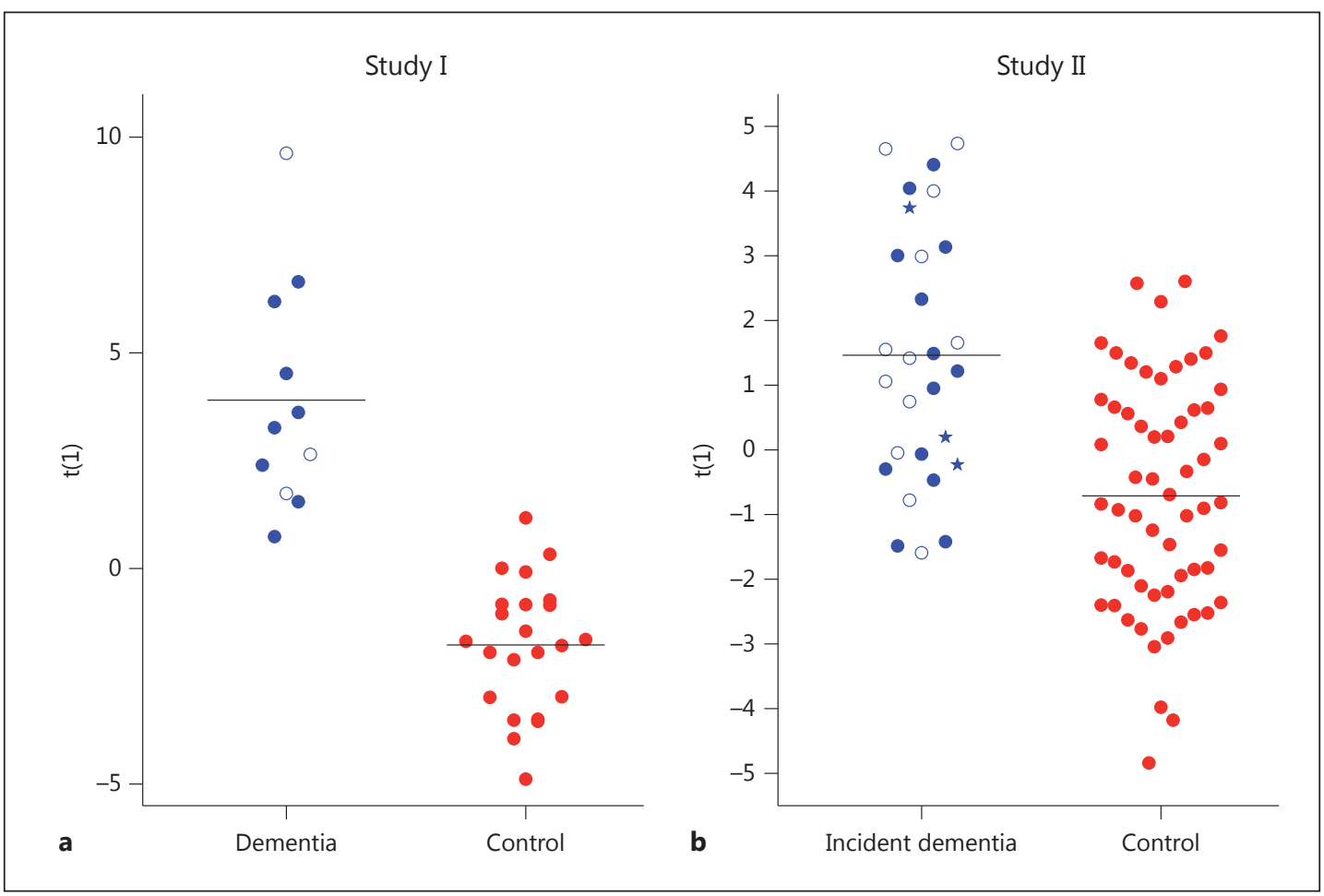

Fig. 2. Predictive OPLS-DA model score [ $\mathrm{t}(1)]$ based on the GC/TOFMS serum metabolite profiles. a Model comparing control and demented patients. Red dots = Controls; blue dots = patients diagnosed with AD; blue circles = patients diagnosed with VaD. $\mathbf{b}$ Model comparing control and incident cases. Red dots = Controls; blue dots = incident $\mathrm{AD}$ cases; blue circles = incident VaD cases; blue pentagrams = incident cases with other diagnosis. The black horizontal lines denote the average of the respective groups in the performed comparisons.

(fig. 3), with a larger percentage of difference between patients and controls (35-55\%) than for incident cases (5-18\%).

The multivariate analysis of saliva samples from study I did not result in a significant model ( $p>0.1)$. Inspection of the chromatograms revealed pronounced variability in the GC/ TOFMS metabolic profiles. Therefore, 35 of 124 samples were selected (without considering group status) on the basis of showing reasonable analytic properties. In the selected samples, 135 peaks (putative metabolites) were detected, which then were quantified in the remaining 89 samples. This resulted in the exclusion of 13 samples, which markedly deviated from the rest. Thereafter, a new model was estimated on the basis of the more restricted set of samples and peaks. The result provided some evidence for a group separation, but more detailed analyses of metabolites that contributed to this model revealed that none of the identified metabolites were significantly different between groups according to ANOVAs (all $p$ values $>0.1$ ). The study II analysis of saliva samples from incident cases and controls did not result in a significant model either.

\section{Discussion}

In this study, multivariate analyses of serum and saliva metabolite profiles of demented patients and incident patients relative to controls were conducted in a sample of individuals who were nondemented and cognitively normal at baseline. Our data show that the serum 


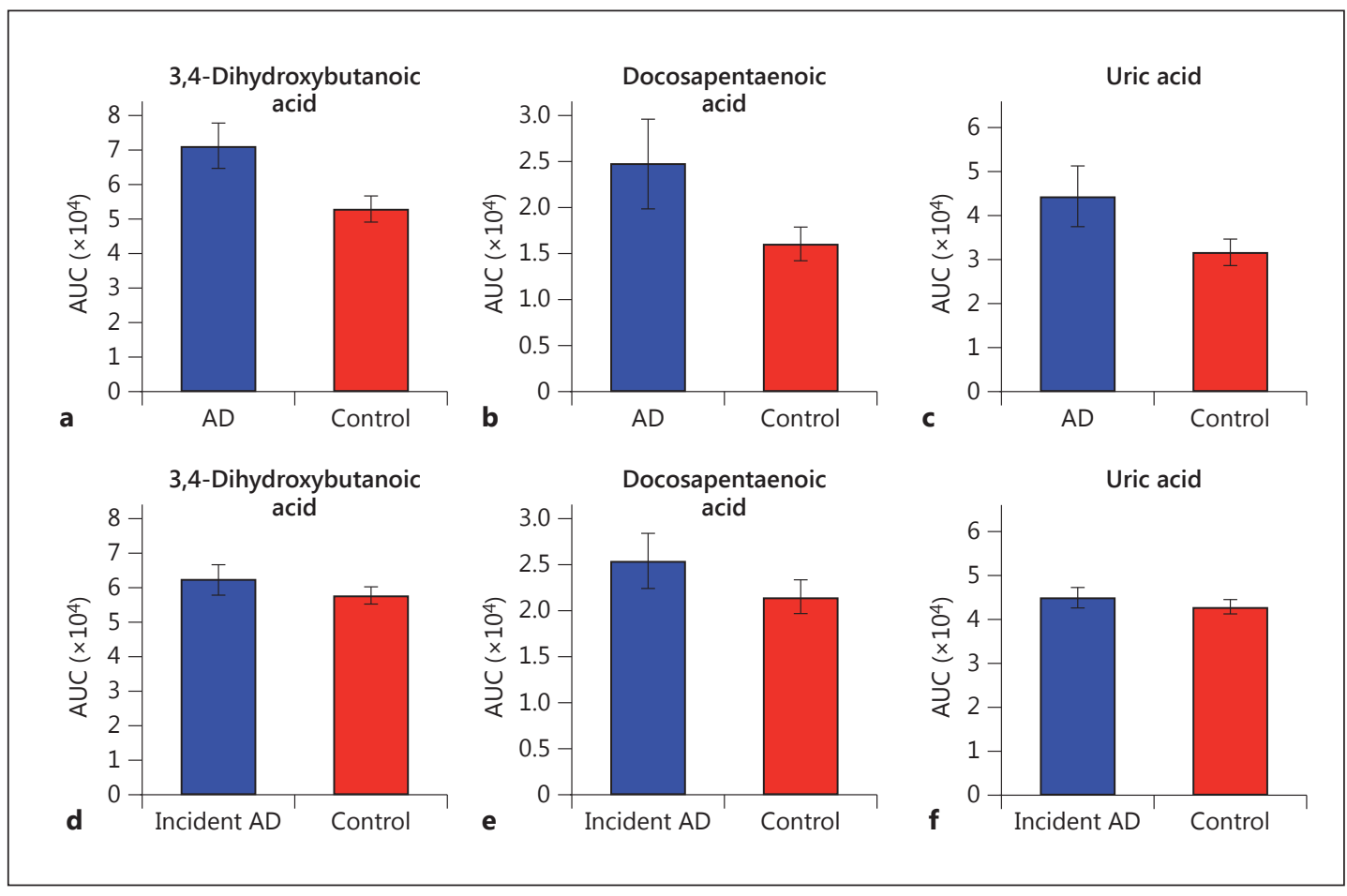

Fig. 3. Mean area under the curve (AUC) for 3 metabolites significantly different between controls and demented patients in study I, and between controls and incident demented cases in study II. a-c Study I (control vs. demented). $\mathbf{d}-\mathbf{f}$ Study II (control vs. incident cases).

metabolite profiles of demented patients differ from those of controls. In addition, differences were seen for serum samples collected at the preclinical stage, up to 5 years preceding dementia. This was found despite the fact that the memory performance of the incident cases and controls was comparable at the preclinical phase. Cognitive decline has been observed several years prior to dementia $[25,26]$, although the consistency of this effect varies across tasks and studies. Here, the participants in study II were apparently in an early stage, with no evidence of subtle cognitive change [27], but still serum metabolomics alterations were detectable.

Prior studies have demonstrated that CSF markers can inform predictions of dementia development $[5,28]$. For serum and saliva, the evidence from previous studies is less conclusive. Zheng et al. [11] found that cognitively healthy older adults could be distinguished from older adults with mild cognitive impairment on the basis of analyses of metabolites identified from saliva samples. Orešič et al. [10] found support that serum metabolite profiles predicted progression to AD. Tsuruoka et al. [12] compared the metabolite profiles among various dementia groups and healthy controls and found no clear group separation for saliva samples but did find significant differences for serum samples. Several factors may affect the usefulness of saliva samples, including those related to contamination, collection procedure, and subsequent storage of samples. The present findings converge with those of Tsuruoka et al. [12] by suggesting that serum is more suitable than saliva for biomarker discovery in dementia.

We found that three serum metabolites (3,4-dihydroxybutanoic acid, docosapentaenoic acid, uric acid) jointly contributed to the separation between demented patients and controls and to the prediction of conversion to dementia. Dihydroxybutanoic acid has previously been 
identified as the major contributor to a model predicting progression to AD from mild cognitive impairment based on serum metabolomic profiles [10]. In that study, 2,4-dihydroxybutanoic acid was upregulated in dementia progressors, similar to what was observed here, which may be indicative of a role of hypoxia in the early dementia pathogenesis. Docosapentaenoic acid was upregulated in a previous study of mild cognitive impairment [29] and also at the presymptomatic stage in a lipidomic analysis of brain tissues and plasma from a mouse model of AD [30], which converges with the present observations. Uric acid has previously been examined in relation to memory and dementia with mixed findings [31, 32]. Consistent with the present observations, high circulating uric acid levels were associated with dementia in a population-based study [33], with low cognitive performance [34], and with increased risk of cognitive decline among older adults [35]. Thus, our observations of upregulation of metabolites for demented patients and incident cases confirm and extend previous findings.

The majority of the demented patients were diagnosed with $\mathrm{AD}$, but there was also a sizable proportion of $\mathrm{VaD}$ ( $25 \%$ in study I, $39 \%$ in study II). We found no evidence for differentiation between $\mathrm{AD}$ and $\mathrm{VaD}$ in the plots from the multivariate analyses (fig. 2). This finding is in line with demonstrations of marked similarities in CSF biomarker profiles between AD and $\mathrm{VaD}$ dementia subgroups [36]. Thus, the results indicate that the observed changes in serum metabolites hold for both $\mathrm{AD}$ and $\mathrm{VaD}$. However, a main limitation in this context is the low number of cases, which not only impacted the possibility to compare different dementia subgroups but also generally reduced the statistical power, in particular in study I. The number of included cases was restricted by the inclusion criterion of having both serum and saliva samples accessible. Future studies of this cohort may, in view of the present results, focus on the serum samples only, thereby increasing the number of available patients and in turn the power to detect additional metabolites that contribute to a separation between demented patients and controls.

In conclusion, the positive findings from the present serum metabolomics analyses, together with other recent findings [37], indicate that the minimally invasive method of blood sampling can make an important contribution to the early prediction of conversion to dementia.

\section{Acknowledgements}

This study was supported by the Erling-Persson Foundation, Knut and Alice Wallenberg Foundation, The Swedish Science Council, Torsten and Ragnar Söderberg's Foundation, and King Gustaf V's and Queen Victoria's Foundation. We thank Dr. Maria Josefsson (Umeå University, Sweden) for assisting in the analyses of memory change over time, and Prof. Kaj Blennow for comments on a previous version.

\section{References}

1 Allan CL, Sexton CE, Welchew D, Ebmeier KP: Imaging and biomarkers for Alzheimer's disease. Maturitas 2010;65:138-142.

-2 Leal SL, Yassa MA: Perturbations of neural circuitry in aging, mild cognitive impairment, and Alzheimer's disease. Ageing Res Rev 2013;12:823-831.

-3 Nordberg A: Molecular imaging in Alzheimer's disease: new perspectives on biomarkers for early diagnosis and drug development. Alzheimers Res Ther 2011;3:34.

4 Blennow K, Hampel H: CSF markers for incipient Alzheimer's disease. Lancet Neurol 2003;2:605-613.

-5 Mattsson N, Rosén E, Hansson O, Andreasen N, Parnetti L, Jonsson M, et al: Age and diagnostic performance of Alzheimer disease CSF biomarkers. Neurology 2012;78:468-476. 
6 Moghekar A, Li S, Lu Y, Li M, Wang M-C, Albert M, et al: CSF biomarker changes precede symptom onset of mild cognitive impairment. Neurology 2013;81:1753-1758.

7 Jack CR, Knopman DS, Jagust WJ, Petersen RC, Weiner MW, Aisen PS, et al: Tracking pathophysiological processes in Alzheimer's disease: an updated hypothetical model of dynamic biomarkers. Lancet Neurol 2013; 12:207-216.

-8 Roher AE, Esh CL, Kokjohn TA, Castaño EM, Van Vickle GD, Kalback WM, et al: Amyloid beta peptides in human plasma and tissues and their significance for Alzheimer's disease. Alzheimers Dement 2009;5:18-29.

-9 Bennett DA: Plasma multianalyte profiling in mild cognitive impairment and Alzheimer disease. Neurology 2013;80:690.

10 Orešič M, Hyötyläinen T, Herukka S-K, Sysi-Aho M, Mattila I, Seppänan-Laakso T, et al: Metabolome in progression to Alzheimer's disease. Transl Psychiatry 2011;1:e57.

11 Zheng J, Dixon RA, Li L: Development of isotope labeling LC-MS for human salivary metabolomics and application to profiling metabolome changes associated with mild cognitive impairment. Anal Chem 2012;84: 10802-10811.

12 Tsuruoka M, Hara J, Hirayama A, Sugimoto M, Soga T, Shankle WR, et al: Capillary electrophoresis-mass spectrometry-based metabolome analysis of serum and saliva from neurodegenerative dementia patients. Electrophoresis 2013;34:2865-2872.

-13 Ibáñez C, Simó C, Martín-Álvarez PJ, Kivipelto M, Winblad B, Cedazo-Mínguez A, Cifuentes A: Toward a predictive model of Alzheimer's disease progression using capillary electrophoresis-mass spectrometry metabolomics. Anal Chem 2012;84:8532-8540.

14 Nilsson L-G, Adolfsson R, Bäckman L, de Frias C, Molander B, Nyberg L: Betula: a prospective cohort study on memory, health and aging. Aging Neuropsychol Cogn 2004;11:134-148.

15 McKhann GM, Knopman DS, Chertkow H, Hyman BT, Jack CR, Kawas CH, et al: The diagnosis of dementia due to Alzheimer's disease: recommendations from the National Institute on Aging-Alzheimer's Association workgroups on diagnostic guidelines for Alzheimer's disease. Alzheimers Dement 2011;7:263-269.

16 American Psychiatric Association: Diagnostic and Statistical Manual of Mental Disorders, ed 4, text revision. Washington, American Psychiatric Association, 2000.

17 Gorelick PB, Scuteri A, Black SE, Decarli C, Greenberg SM, Iadecola C, et al: Vascular contributions to cognitive impairment and dementia: a statement for healthcare professionals from the American Heart Association/ American Stroke Association. Stroke 2011;42:2672-2713.

18 Josefsson M, de Luna X, Pudas S, Nilsson L-G, Nyberg L: Genetic and lifestyle predictors of 15-year longitudinal change in episodic memory. J Am Geriatr Soc 2012;60:2308-2312.

19 Pudas S: Brain Characteristics of Memory Decline and Stability in Aging; thesis, Stockholm, 2013..

-20 Jiye A, Trygg J, Gullberg J, Johansson AI, Jonsson P, Antti H, et al: Extraction and GC/MS analysis of the human blood plasma metabolome. Anal Chem 2005;77:8086-8094.

-21 Jonsson P, Johansson AI, Gullberg J, Trygg J, Jiye A, Grung B, et al: High-throughput data analysis for detecting and identifying differences between samples in GC/MS-based metabolomic analyses. Anal Chem 2005;77: 5635-5642.

22 Thysell E, Chorell E, Svensson M, Jonsson P, Antti H: Validated and predictive processing of gas chromatography-mass spectrometry based metabolomics data for large scale screening studies, diagnostics and metabolite pattern verification. Metabolites 2012;2:796-817.

23 Schauer N, Steinhauser D, Strelkov S, Schomburg D, Allison G, Moritz T, et al: GC-MS libraries for the rapid identification of metabolites in complex biological samples. FEBS Lett 2005;579:1332-1337.

24 Redestig H, Fukushima A, Stenlund H, Moritz T, Arita M, Saito K, et al: Compensation for systematic crosscontribution improves normalization of mass spectrometry based metabolomics data. Anal Chem 2009;81: 7974-7980.

-25 Bäckman L, Small B, Fratiglioni L: Stability of the preclinical episodic memory deficit in Alzheimer's disease. Brain 2001;124:96-102.

26 Amieva H, Jacqmin-Gadda H, Orgogozo J-M, Le Carret N, Helmer C, Letenneur L, et al: The 9 year cognitive decline before dementia of the Alzheimer type: a prospective population-based study. Brain 2005;128:10931101.

27 Sperling RA, Aisen PS, Beckett LA, Bennett DA, Craft S, Fagan AM, et al: Toward defining the preclinical stages of Alzheimer's disease: recommendations from the National Institute on Aging-Alzheimer's Association workgroups on diagnostic guidelines for Alzheimer's disease. Alzheimers Dement 2011;7:280-292.

-28 Gustafson DR, Skoog I, Rosengren L, Zetterberg H, Blennow K: Cerebrospinal fluid beta-amyloid 1-42 concentration may predict cognitive decline in older women. J Neurol Neurosurg Psychiatry 2007;78:461-464.

29 Milte CM, Sinn N, Street SJ, Buckley JD, Coates AM, Howe PRC: Erythrocyte polyunsaturated fatty acid status, memory, cognition and mood in older adults with mild cognitive impairment and healthy controls. Prostaglandins Leukot Essent Fatty Acids 2011;84:153-161.

-30 Tajima Y, Ishikawa M, Maekawa K, Murayama M, Senoo Y, Nishimaki-Mogami T, et al: Lipidomic analysis of brain tissues and plasma in a mouse model expressing mutated human amyloid precursor protein/tau for Alzheimer's disease. Lipids Health Dis 2013;12:68.

-31 Euser SM, Hofman A, Westendorp RGJ, Breteler MMB: Serum uric acid and cognitive function and dementia. Brain 2009;132:377-382. 
-32 Kim T, Pae C, Yoon S, Jang W, Lee NJ, Kim J, et al: Decreased plasma antioxidants in patients with Alzheimer's disease. Int J Geriatr Psychiatry 2006;21:344-348.

33 Ruggiero C, Cherubini A, Lauretani F, Bandinelli S, Maggio M, Di Iorio A, et al: Uric acid and dementia in community-dwelling older persons. Dement Geriatr Cogn Disord 2009;27:382-389.

34 Verhaaren BFJ, Vernooij MW, Dehghan A, Vrooman HA, de Boer R, Hofman A, et al: The relation of uric acid to brain atrophy and cognition: the Rotterdam Scan Study. Neuroepidemiology 2013;41:29-34.

-35 Schretlen DJ, Inscore AB, Jinnah HA, Rao V, Gordon B, Pearlson GD: Serum uric acid and cognitive function in community-dwelling older adults. Neuropsychology 2007;21:136-140.

-36 Kaerst L, Kuhlmann A, Wedekind D, Stoeck K, Lange P, Zerr I: Cerebrospinal fluid biomarkers in Alzheimer's disease, vascular dementia and ischemic stroke patients: a critical analysis. J Neurol 2013;260:2722-2727.

-37 Mapstone M, Cheema AK, Fiandaca MS, Zhong X, Mhyre TR, Macarthur LH, et al: Plasma phospholipids identify antecedent memory impairment in older adults. Nat Med 2014;20:415-418. 